\title{
Deep Learning Enabled Predictive Model for P2P Energy Trading in TEM
}

\author{
Pudi Sekhar $^{1}$, T. J. Benedict Jose ${ }^{2}$, Velmurugan Subbiah Parvathy ${ }^{3}$, E. Laxmi Lydia ${ }^{4}$, \\ Seifedine Kadry ${ }^{5}$, Kuntha Pin $^{6}$ and Yunyoung Nam ${ }^{7}$,*
}

\author{
${ }^{1}$ Department of Electrical and Electronics Engineering, Vignan's Institute of Information Technology (Autonomous), \\ Visakhapatnam, 530049, India \\ ${ }^{2}$ Department of Computer Applications, Government Arts \& Science College, Kanyakumari, 629401, India \\ ${ }^{3}$ Department of Electronics and Communication Engineering, Kalasalingam Academy of Research and Education, \\ Krishnankoil, 626126, India \\ ${ }^{4}$ Department of Computer Science and Engineering, Vignan's Institute of Information Technology (Autonomous), \\ Visakhapatnam, 530049, India \\ ${ }^{5}$ Department of Applied Data Science, Noroff University College, Kristiansand,Norway \\ ${ }^{6}$ Department of ICT Convergence, Soonchunhyang University, Korea \\ ${ }^{7}$ Department of Computer Science and Engineering, Soonchunhyang University, Korea \\ *Corresponding Author: Yunyoung Nam. Email: ynam@sch.ac.kr \\ Received: 27 July 2021; Accepted: 27 August 2021
}

\begin{abstract}
With the incorporation of distributed energy systems in the electric grid, transactive energy market (TEM) has become popular in balancing the demand as well as supply adaptively over the grid. The classical grid can be updated to the smart grid by the integration of Information and Communication Technology (ICT) over the grids. The TEM allows the Peerto-Peer (P2P) energy trading in the grid that effectually connects the consumer and prosumer to trade energy among them. At the same time, there is a need to predict the load for effectual P2P energy trading and can be accomplished by the use of machine learning (DML) models. Though some of the short term load prediction techniques have existed in the literature, there is still essential to consider the intrinsic features, parameter optimization, etc. into account. In this aspect, this study devises new deep learning enabled short term load forecasting model for P2P energy trading (DLSTLF-P2P) in TEM. The proposed model involves the design of oppositional coyote optimization algorithm (OCOA) based feature selection technique in which the OCOA is derived by the integration of oppositional based learning (OBL) concept with COA for improved convergence rate. Moreover, deep belief networks (DBN) are employed for the prediction of load in the P2P energy trading systems. In order to additional improve the predictive performance of the DBN model, a hyperparameter optimizer is introduced using chicken swarm optimization (CSO) algorithm is applied for the optimal choice of DBN parameters to improve the predictive outcome. The simulation analysis of the proposed DLSTLF-P2P is validated using the UK Smart Meter dataset and the obtained outcomes demonstrate the superiority of the DLSTLF-P2P technique with the maximum training, testing, and validation accuracy of $90.17 \%, 87.39 \%$, and $87.86 \%$.
\end{abstract}


Keywords: Energy trading; distributed systems; power generation; load forecasting; deep learning; peer-to-peer

\section{Introduction}

The overview of distributed energy resource (DER) in conventional power system provides a way of creating energy. It carries several benefits, like decreasing environment pollution, lower the power cost of DERs holders, minimize cost, etc. [1]. Furthermore, DERs owners could transform from consumer to prosumer by trading their excess energy production to other users and grids. For effective energy managing and incentivizing the DERs owner appropriately, a novel market structure named Transactive Energy Market (TEM) was developed [2]. TEM inspires small-scale consumers as well as generators for joining in traditional power market to buy, produce and sell energies. The traditional grid, in which electricity is produced mainly in large central producers, transported to the distribution centres, and later shared to the end user, is updating to a smart grid via combining Information and Communication Technology (ICT) through the grid. TEM allows Peer-to-Peer (P2P) electricity trading in the grid [3] that creates a link among prosumers and consumers to trade energy with one another. In the P2P electricity trading, energy from smaller scale DER in factories, offices, dwellings, and so on, is traded amongst neighboring consumers and prosumers. The advantages of P2P electricity trading are twofold as the prosumer gets financial benefits via trading their surplus renewable production, and consumers purchase electrical energy at a lower level from their peer rather than the grid.

Furthermore, executing TEM in the smart grid allows flexible systems in which consumers or prosumers could adapt utilization/generation patterns of DERs according to the request of distinct entities like network service providers and retailers in cases of urgency [4]. Also, TEM assists grid operators to address the grids increasing complexities and coordinate energy utilization and generation depend on electricity price signal. But, TEM execution in electrical grid has various problems because of the irregular energy production of DER as present electrical energy markets could not respond in real-time to the arbitrary production from DER. Moreover, electricity price in the markets is frequently established on a national level that doesn't deliberate surplus of supply/local energy shortage.

Based on the idea of transactive energy, a new suggestion to engage prosumers in electricity market is $\mathrm{P} 2 \mathrm{P}$ trading method. It denotes the direct electricity trading amongst prosumers and consumers in distribution network, i.e., evolved on the basis of P2P economy idea. Various frameworks could be executed for P2P energy trading, like full P2P, hybrid structure, and community based. Market design to trade among performers within a community was extensively deliberated in the survey utilizing microgrid (MG) as a standard for community dependent trading [5]. The electricity distribution method among peers is projected by a dynamic internal price. A collective electricity dispatch approach for interactions amongst MGs. Nested transactive concepts are proposed for modelling a distribution grid with various virtual MGs, in which every MG could act as a market. Direct electricity trading's amongst many MGs is developed as a widespread Nash bargaining problem for maximizing social welfare [6]. Currently, novel designs for complete P2P electricity trading were projected to allow prosumers for engaging directly in bilateral energy trading. A novel market design on the basis of two-sided contract networks for trading electricity in advance and realtime markets, in which a

distributed price alteration is utilized for clearing markets. A P2P electricity trading's among electric vehicles (EVs) are presented for reducing the impacts of EV charging on peak power. A complete 
P2P market framework for bilateral electricity trading amongst market performers according to their preference [7].

This study devises new deep learning enabled short term load forecasting model for P2P energy trading (DLSTLF-P2P) in TEM. The proposed model involves the design of oppositional coyote optimization algorithm (OCOA) based feature selection approach in which the OCOA is a derivative by the integration of oppositional based learning (OBL) concept with COA for improved convergence rate. Followed by, deep belief networks (DBN) is employed for the prediction of load in the P2P energy trading systems. To raise the predictive outcome of the DBN model, a hyperparameter optimizer is presented using chicken swarm optimization (CSO) technique. The integration of the OCOA based feature selection and CSO based hyperparameter optimization shows the novelty of the study. A widespread experimental analysis is executed on the UK Smart Meter dataset and inspect the outcomes under various aspects.

The rest of the paper is organized as follows. Section 2 offers the brief literature review and Section 3 introduces the proposed model. Then, Section 4 provides the result analysis and Section 5 draws the conclusion.

\section{Literature Review}

Mohamed et al. [8] emphases on the online detection False Data Injection Attack (FDIA) that attempts to interrupt the trends of optimum P2P energy trading in the stochastic scenario. Chen et al. [9] incorporate Deep Reinforcement Learning (DRL) method and realistic P2P energy trading method for addressing decision making problems for MGs in the local energy markets. Firstly, an hour in advance P2P energy trading method with a group of critical physical constraints is made. Later, the decision making procedure of energy trading is constructed as a Markov decision procedure. Besides, an adapted deep Q-network (DQN) method assists the MG in using their assets and create improved strategy. Lastly, they select several real time electricity datasets for performing the simulation.

Qiu et al. [10] categorizes the contributing prosumer to many clusters regarding their collection of DER and analyzing their trading decision in a simulative P2P trading environment. The later applies the mid-market rate (MMR) local pricing method for enabling energy trading between penalizes and prosumers the participation to the scheme demand peak of all prosumers. They develop the P2P trading issue as a multiagent coordination issue and proposed a new multi-agent DRL (MADRL) technique for addressing it. The presented technique is allows to speed up the training speed by learned policies and sharing experiences amongst each agent in all clusters, however, it also sustains the policy diversity among multiple clusters. Jamil et al. [11] proposed a block chain based prediction energy trading environment for day in advance controlling, generation scheduling of distributed energy resource, and providing real world support. The projected blockchain based environment contains 2 components; block chain based energy trading and smart contract enabled prediction analytic models. The smart contracts based prediction analytic model's aim is to construct a predictive method on the basis of past energy consuming data for predicting short term consumption of energy.

Khorasany et al. [12] proposed a hybrid energy trading system for P2P energy trading in transactive energy market. Market performers could take part in various markets, involving traditional trading with the grid, trading with neighborhood areas, and local markets. Depending upon the heterogeneous preference of performers of every community, every local market contains distinct prices, i.e., unlike trading with the grid and market price for neighbourhood trading. A distributed market clearing method is proposed, which integrates coordination between various markets. Ullah et al. [13] presented a privacy-preserving distributed dynamic pricing approach for P2P TEM in the 
smart grids with Fast Alternating Direction Method of Multipliers (F-ADMM) approach. The results show that the approach converged rapidly and simplifies easier execution. Furthermore, a closed form solution for a P2P transactive energy scheme was proposed, that speeds up the entire computational time.

Yan et al. [14] proposed a 2 levels network constrained P2P TEM for MGs, that permits MG for trading energy with one another. At low level, P2P transactive energy is applied to MGs for trading energy with one another. Zheng et al. [15] proposed an equilibrium method of a P2P transactive energy market. Each contributor seeks the maximal own interests, with the option of providing energy from or to another peer through distinct buses of the distribution network. These criteria are attained by integrating the Karush Kuhn Tucker condition of each problem of separate participants.

Kim et al. [16] proposed a novel trading calculation condition which considers this factor by determining gain as the amount of the profits from 4 modules: trading, electricity bill, virtual loss, and electric energy stored in the energy storage system. For the projected automated P2P energy trading method, they adapt a long-term delayed reward technique which calculates the delayed reward appears monthly by creating the end point.

In Wongthongtham et al. [17], blockchain technique for P2P energy trading and its implication is examined, particularly considering the 'trilemma': decentralization, scalability, and security. P2P energy trading is the emphasis of this work that eventually suggests a blockchain scalability solution. These solutions are empirically modeled with data gathered in experimental analysis. Ferrag et al. [18] devised a DL and blockchain enabled energy architecture called DeepCoin. The DeepCoin architecture using 2 systems, a block chain based system, and a DL based system. For preventing smart grids attack, the presented method creates the generation of blocks through hash functions and short signatures.

\section{The Proposed Model}

In this study, a novel DLSTLF-P2P technique is developed to derive effectual load forecasting model for P2P energy trading in TEM. The DLSTLF-P2P technique encompasses several processes namely preprocessing, OCOA based feature selection, DBN based classification, and CSO based hyperparameter optimization. The working of these processes is elaborated in the succeeding sections.

\subsection{Feature Selection Using OCOA Technique}

The OCOA technique examines the data and chooses an optimal selection of feature subsets. $\mathrm{COA}$ is a recently developed metaheuristic technique which is based on the adaptable characteristics of the coyotes and also, they face interchange action. It has a fascinating approach to balancing among exploration and exploitation. It begins with $N_{P}$ population size and $N_{c}$ coyote count as the candidate solution. The COA defines the social nature of the coyotes as the cost function and can be defined in Eq. (1):

$S O C_{c}^{p, t}=x=\left[x_{1}, x_{2}, \ldots, x_{D}\right]$

where $c$ defines the number and $p$ labels the group and $t$ denote the simulation time for the designing parameters. Firstly, few arbitrary coyotes are produced as the solution candidates that exist in the searching area, as given below.

$S O C_{c, j}^{p, t}=L B_{j}+\eta \times\left(U r_{j}-L r_{j}\right)$ 
where $\eta \in[0,1]$ indicates an arbitrary value and $L r_{j}$ and $U r_{j}$ denotes the minimum and maximum boundary of the jth parameter in the searching area. The cost function of the coyotes can be defined as follows.

$\operatorname{obj}_{c}^{p, t}=f\left(S O C_{c, j}^{p, t}\right)$

The COA upgrades the location of the groups in a random way. In addition, the candidate can upgrade the location once it is left from the group, and the leaving process is formulated based on the probability as given below [19].

$P_{1}=0.05 \times N_{c}^{2}$

where $c \leq \sqrt{200}, P_{1}>1$. The coyote count in a group should be restricted to 14 in order to improve the algorithmic diversity, i.e., cultural interaction between coyotes. The optimal solution of the iterations are treated as the alpha coyotes, as provided in Eq. (5):

$\alpha^{p, t}=\operatorname{soc}_{c}^{p, t}$ for $\min o b j_{c}^{p, t}$

The general features of the coyotes for the culture transformation is defined below:

$c u l_{j}^{p, t}=\left\{\begin{array}{l}R^{p, t}{ }_{\frac{N_{C}+1}{2}, j}, N_{c} \text { is odd number } \\ \frac{1}{2}\left(R^{p, t} \frac{N_{C}, j}{2}+R^{p, t} \frac{N_{C}}{2}+1, j\right) 0 . W .\end{array}\right.$

where, $R^{p, t}$ computes the coyote, social condition grade for group number $p$ at time $t$ for the parameter $j$.

The COA treats the coyote lifecycle that is an integration of environment factors and social characteristics of the parent coyote. The coyote's lifecycle can be represented using Eq. (7):

$B l e_{j}^{p, f}=\left\{\begin{array}{l}s o c_{r_{1}, \mathrm{j}}^{p, t}, r_{j}<p r_{s} \text { or } j=j_{1} \\ \operatorname{soc}_{r_{2} j}^{p, t}, r_{j} \geq p r_{s}+p r_{a} \text { or } j=j_{2} \\ \sigma_{j}, O . W .\end{array}\right.$

where $r_{j} \in[0,1]$ denotes arbitrary values and $r_{2}$ indicates an arbitrary coyote in the group $p, \sigma_{j}$ defines an arbitrary value in the design variable limits, $j_{1}$ and $j_{2}$ are arbitrary design variables, and $p r_{a}$ and $p r_{s}$ signify the association and scatter probability which declares the coyote cultural diversity from the group. The $p r_{a}$ and $p r_{s}$ can be mathematically defined here's:

$p r_{s}=\frac{1}{d}$

$\operatorname{Pr}_{a}=\frac{1}{2}(1-p r)$

where $d$ denotes the variable dimensions. The pseudocode of the balancing procedure of coyote's lifecycle is provided here.

Compute $i$ and $\omega$

if $i=1$

Ble survives and coyote in $\omega$ deceases

else if $i>1$

Ble survives and eldest coyote in $\omega$ expires 
else

Ble deceases

End if

where $i$ denotes the coyote count in the group, $\omega$ computes the worse outcome of the coyote, and the probability of decreasing for Ble is found to be $10 \%$. The cultural conversion amongst the groups can be represented using $\delta_{1}$ and $\delta_{2}$ as given below:

$\delta_{1}=\alpha^{p, t}-\operatorname{soc}_{c r 1}^{p, t}$

$\delta_{2}=\mathrm{cul}^{p, t}-\operatorname{soc}_{c r 2}^{p, t}$

where, $\delta_{1}$ signifies the cultural variations among the leader (alpha) and chosen coyote ( $\left.c r 1\right)$ and $\delta_{2}$ defines the culture variance among group culture trending and chosen coyote ( $c r 2)$. To update the social characteristics depending upon the leader and the group impact, Eq. (12) is used.

$n \operatorname{soc}_{c}^{p, t}=\operatorname{soc}_{c}^{p, t}+r_{1} \times \delta_{1}+r_{2} \times \delta_{2}$

where $r_{1}$ and $r_{2}$ represents arbitrary numbers. With the update conditions, the new cost is attained using Eq. (14):

$\operatorname{nobj}_{c}^{p, t}=f\left(\operatorname{nsoc}_{c}^{p, t}\right)$

$\operatorname{soc}_{c}^{p, t+1}=\left\{\begin{array}{l}n s o c_{c}^{p, t}, n o b j_{c}^{p, t}<o b j_{c}^{p, t} \\ \operatorname{soc}_{c}^{p, t}, O . W .\end{array}\right.$

A vital characteristic of these approaches is the capability to escape from the local optima.

The OCOA technique is derived by the use of OBL concept into the COA technique for raising the convergence rate. For improving the efficiency of COA technique, Opposition Based Learning (OBL) was comprised of SSO technique to increase the convergence rate. It works by exploring both ways in exploring areas like original and opposite solutions. The opposite number $x$ is expressed as real number in the interval $x \in[l b, u b]$. The opposite number of $x$ is demonstrated as $\tilde{x}$ :

$\tilde{x}=l b+u b-x$

In order to generalize, every searching agent and opposite solution is determined utilizing Eqs. (16) and (17):

$x=\left[x_{1}, x_{2}, x_{3}, \ldots x_{D}\right]$

$\tilde{x}=\left[\tilde{x}_{1}, \tilde{x}_{2}, \tilde{x}_{3}, \ldots, \tilde{x}_{D}\right]$

The values of all elements in $\tilde{x}$ is referred to as (18):

$\tilde{x}_{j}=l b_{j}+u b_{j}-x_{j}$ where $j=1,2,3, \ldots, D$

If the fitness value $f(\tilde{x})$ of opposite solution is optimum than $f(x)$ of their actual solutions $x$, afterward $x=\tilde{x}$; else $x=x$.

The existence of repetitive features can affect learning performance. The FS process ensures the relativeness of the features which comprise a dataset and eradicating the features which do not helps to achieve effective outcomes. The features chosen by the FS model can be defined as a $N$ sized vector where $N$ denotes the feature count in the dataset in which each place of the vector considers two 
values ( 0 or 1$)$. The value of 0 denotes the feature is unselected and 1 indicates that the feature is chosen. The transfer function (TF) represents the possibility of modifying a location vector component from $0-1$ and vice versa. The TF affects the outcome of the FS model at the time of searching the optimal features. It avoids the local optimum problem and balances exploration and exploitation [20]. In OCOA, the social state of the coyotes corresponds to binary values by the use of $v$-shaped TF as given below:

$V\left(n e w_{-} \operatorname{Soc}_{c}^{p, t}\right)=\left|\frac{n e w_{-S 0 c_{c}^{p, t}}}{\sqrt{1+\left(n e w_{-} S O c_{c}^{p, t}\right)^{2}}}\right|$,

where $n e w_{-} s o c_{c}^{p, t}$ resembles the upgraded social stage vector with the consideration of the continuous values. The OCOA technique makes use of the s-shaped TF:

$V(x)=\left|\frac{1}{\sqrt{1+e^{-a(x-c)}}}\right|$,

where $x$ signifies the location vector of the upgraded individual with the consideration of the continuous values.

\subsection{Load Prediction Using DBN Model}

The feature subsets from the OCOA technique are fed into the DBN model for appropriate prediction of load. DBN is a probabilistic generative model which contains a stacking of multiple layers of Restricted Boltzmann Machines (RBM) in which everyone includes a layer of visible and hidden layers. The DBN model undergoes extraction of a deep hierarchical representation of the input data by the use of greedy layer wise procedure. Once the layers of the RBM have undergone training, the representation of the earlier hidden layer is fed as input to the succeeding hidden layer. The structure of DBN is depicted in Fig. 1 . The DBN model with $l$ hidden layers includes $l$ weight matrix: $W^{(1)}, \ldots, W^{(l)}$. It additionally includes $l+1$ bias vectors: $b^{(0)}, \ldots, b^{(l)}$ with $b^{(0)}$ offering the bias for the visible layer. The probability distribution of the DBN model can be defined as follows.

$P\left(h^{(l)}, h^{(l-1)}\right) \propto \exp \left(b^{(l)^{T}} h^{(l)}+b^{(l-1)^{T}} h^{(l-1)}+h^{(l-1)^{T}} W^{(l)} h^{(l)}\right)$,

$P\left(h_{i}^{(k)}=1 \mid h^{(k+1)}\right)=\sigma\left(b_{i}^{(k)}+W_{:, i}^{(k+1)^{T}} h^{(k+1)}\right) \forall i, \forall k \in 1, \ldots, l-2$,

A 2-layer DBN stacked through 2 RBMs holds a set of visible layers and 2 layers of hidden layers. If $h^{(1)}$ and $h^{(2)}$ denotes the state vectors of the hidden layer, $v$ represents state vector of the visible layer, $W^{(1)}$ and $W^{(2)}$ indicates the matrix of the symmetrical weight, $b^{(1)}$ and $b^{(2)}$ signifies bias vectors of the hidden layers, and $b^{(0)}$ is the bias vectors of the visible layer.

$P\left(v_{i}=1 \mid h^{(1)}\right)=\sigma\left(b_{i}^{(0)}+W_{i}^{(1)^{T}} h^{(1)}\right) \forall i$.

When the real valued visible layers have existed, then apply $v$ with $\beta$ diagonal for manageability [21].

$v \sim N\left(b^{(0)}+W^{(1)^{T}} h^{(1)}, \beta^{-1}\right)$,

$\sigma(x)=1 /(1+\exp (-x))$.

$h^{(1)}=\sigma\left(b^{(1)}+v^{T} W^{(1)}\right)$, 
$h^{(l)}=\sigma\left(b_{i}^{(l)}+h^{(l-1)^{T}} W^{(l)}\right), \forall l \in 2, \ldots, m$

The weight from the trained DBN can be optimally tuned to enhance the efficiency of the entire network.

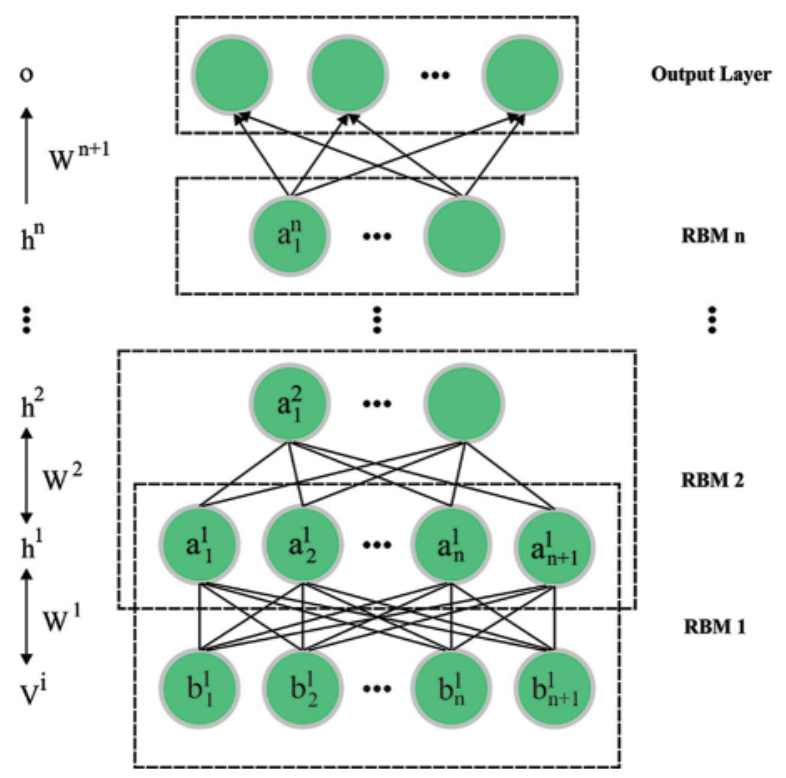

Figure 1: Framework of DBN

\subsection{Hyperparameter Optimization Using CSO Algorithm}

The CSO algorithm is employed as a hyperparameter optimization to effectually tune the parameters involved in the DBN model. The CSO algorithm considers the optimization issue as the procedure of searching food by chickens. The entire CS (CS) is partitioned into various flocks of chicken including a cock, hens, and chicks. There is a rivalry amongst every CS and the optimal cluster individual is stained by the competition. This process can be simplified as given below.

1. Every CS includes several sub-CSs everyone includes a cock, hens, and chicks.

2. The CS partitions different sub-CSs and compute the FV of the individual. The ones with optimum fitness value (FV) is chosen as cock and it becomes the leader of the CS. The chicken with least FV becomes chick and the remining ones become hens. The hen generally follows a cock arbitrarily and the relativity among the hens as well as chicks are arbitrarily created.

3. The dominance connection, hierarchy, and mother child bond in the CS remains same; chickens regroups and updates role for each $G$ generation.

4. The sub-CS searches food with the cock and the chick looks for food over the hens, and it has the ability to identify the food. The cock, hen, and chick in the CS carry out the optimization processes distinctly.

The individuals in the CS moves based on their rules till optimal location is identified. In the CSO algorithm, the individual count in the flocks is kept as $N$, and the location of each CS individual can be defined as $x_{i, j}(t)$, and it represents that the location attained in the $t-t$ th round of the $i-t h$ flock individuals in the $j-t$ dimension. So, there are different locations for the 3 distinct kinds of chickens 
in the CS optimization. The cock attains the optimal FV in all sub-groups and it finds food effectively [22]. The location of the cock can be upgraded using the following equation.

$x_{i, j}(t+1)=x_{i, j}(t) *\left(1+\operatorname{Rann}\left(0, \sigma^{2}\right)\right)$,

$\sigma^{2}= \begin{cases}1, & f_{i} \leq f_{k}, \\ \exp \left(\frac{f_{k}-f_{i}}{\left|f_{i}\right|+\varepsilon}\right) & f_{i}>f_{k},\end{cases}$

where Ran $n\left(0, \sigma^{2}\right)$ offers an average of 0 and Gaussian distribution arbitrary number with standard deviation $\sigma, \varepsilon$ is tool small, $f_{i}$ and $f_{k}$ indicates the $\mathrm{FV}$ of individuals $i$ and $k$. The location of the hen can be upgraded using Eq. (28):

$x_{i, j}(t+1)=x_{i, j}(t)+c_{1} * \operatorname{ran} *\left(x_{r 1, j}(t)-x_{i, j}(t)\right)+c_{2} * \operatorname{ran} *\left(x_{r 2, j}(t)-x_{i, j}(t)\right)$,

$c_{1}=\exp \left(\frac{\left(f_{i}-f_{r 1}\right)}{a b s\left(f_{1}\right)+\varepsilon}\right)$,

$c_{1}=\exp \left(f_{r 2}-f_{i}\right)$,

where ran denotes the arbitrary number in the range of $0-1$. The location equivalent to the chick can be upgraded using Eq. (29):

$x_{i, j}(t+1)=x_{i, j}(t)+F *\left(x_{m, j}(t)-x_{i, j}(t)\right)$,

where $m$ defines the hen equivalent to the $\mathrm{i}^{\text {th }}$ chick and $F$ is the follow up coefficient. Fig. 2 demonstrates the flowchart of CSO algorithm.

\section{Performance Validation}

The performance of the DLSTLF-P2P technique is examined using the UK Smart Meter dataset [23]. The applied dataset has different attributes such as Household id, Plans used (standard or dynamic time of use), Date and Time, Meter readings (Kwh), and Acorn groups. A comprehensive MAPE analysis of the DLSTLF-P2P technique with other techniques is performed in Fig. 3. The figure reported that the ARIMA technique has offered a worse performance with the training, testing, and validation MAPE of 39.57, 42.65, and 52.09. Also, the AdaBoost technique has demonstrated somewhat decreased training, testing, and validation MAPE of 38.24, 40.43, and 40.23. Then, the gradient boosting model has gained even reduced training, testing, and validation MAPE of 34.46, 31.46, and 36.67. Additionally, the LDA, ANN, SVR, and ARIMA-NN techniques have led to reasonable training, testing, and validation MAPE values. However, the proposed DLSTLF-P2P technique has outpaced the other techniques with the lower training, testing, and validation MAPE of 11.32, 13.89, and 14.99.

A brief MSE analysis of the DLSTLF-P2P approach with other methods is performed in Fig. 4. The figure described that the ARIMA manner has obtainable the least performance with the training, testing, and validation MSE of $0.38,0.29$, and 0.52 . Also, the SVR algorithm has outperformed slightly reduced training, testing, and validation MSE of 0.38, 0.26, and 0.49. Afterward, the ANN algorithm has increased even reduced training, testing, and validation MSE of 0.37, 0.24, and 0.44. Moreover, the AdaBoost, gradient boosting, ARIMA-NN, and LDA manners have led to reasonable training, testing, and validation of MSE values. But, the projected DLSTLF-P2P methodology has outpaced the other algorithms with the minimal training, testing, and validation MSE of 0.12, 0.15, and 0.13. 


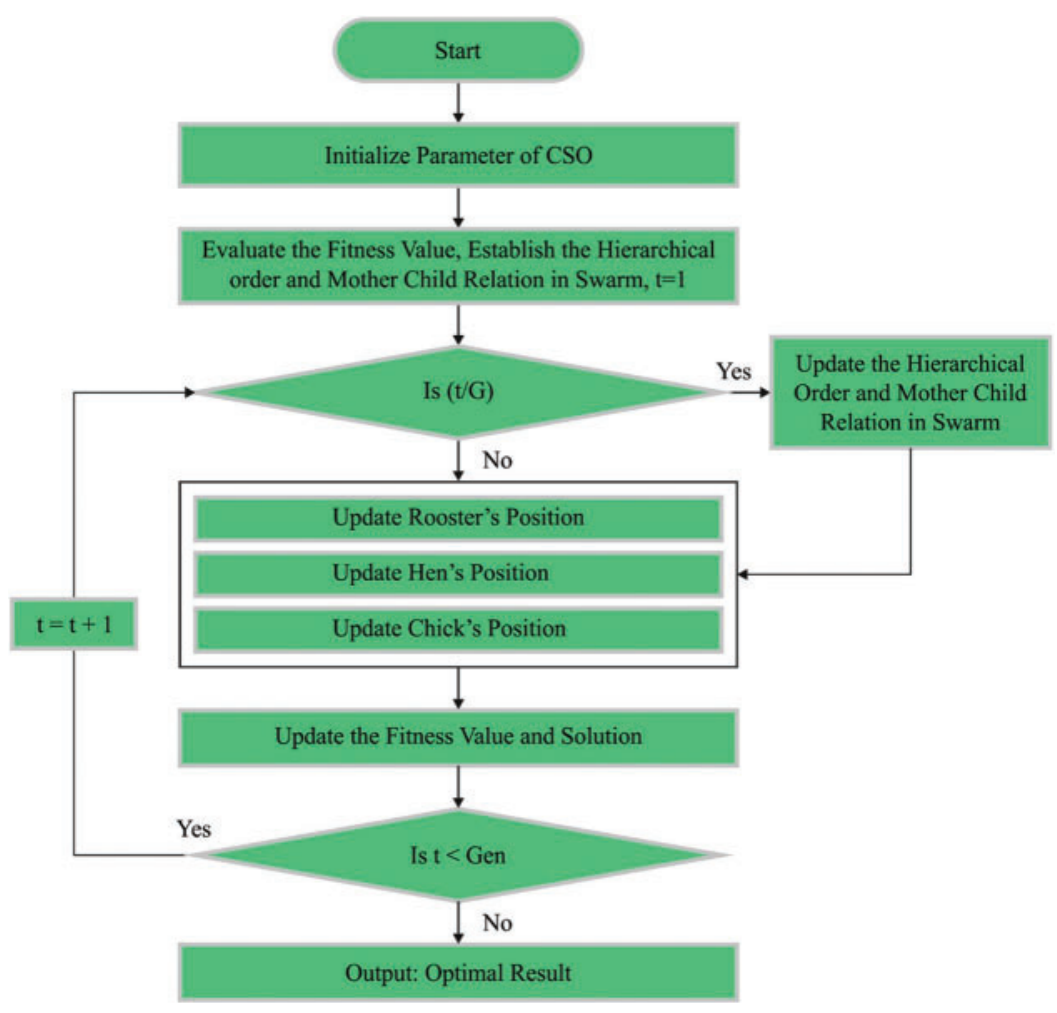

Figure 2: Flowchart of CSO

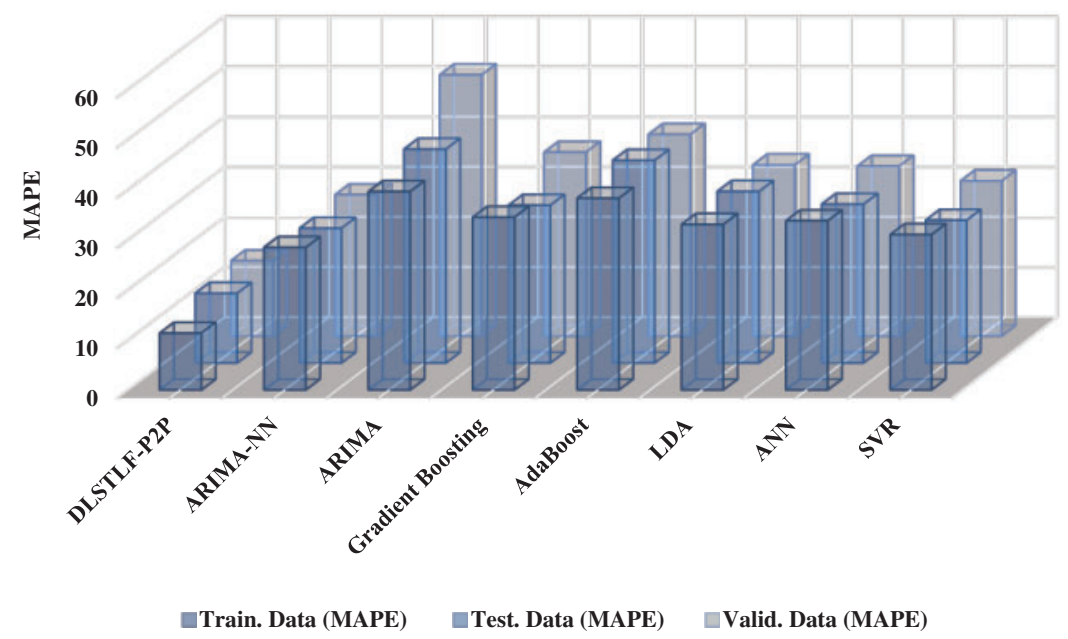

Figure 3: Result analysis of DLSTLF-P2P model interms of MAPE

Detailed accuracy analysis of the DLSTLF-P2P technique with other techniques take place in Tab. 1. The simulation outcomes portrayed that the LDA technique has resulted in poor outcomes with the training, testing, and validation accuracy of $30.38 \%, 40.57 \%$, and $37.39 \%$. In addition, the ARIMA technique has tried to showcase slightly enhanced training, testing, and validation accuracy of $30.51 \%, 20.33 \%$, and $18.07 \%$. Followed by, the gradient boosting model has accomplished even 
increased training, testing, and validation accuracy of $35.65 \%, 40.87 \%$, and $32.78 \%$. Moreover, the AdaBoost, ANN, SVR, and ARIMA-NN techniques have resulted in moderate training, testing, and validation accuracy values. However, the proposed DLSTLF-P2P technique has outperformed the other techniques with the maximum training, testing, and validation accuracy of $90.17 \%, 87.39 \%$, and $87.86 \%$.

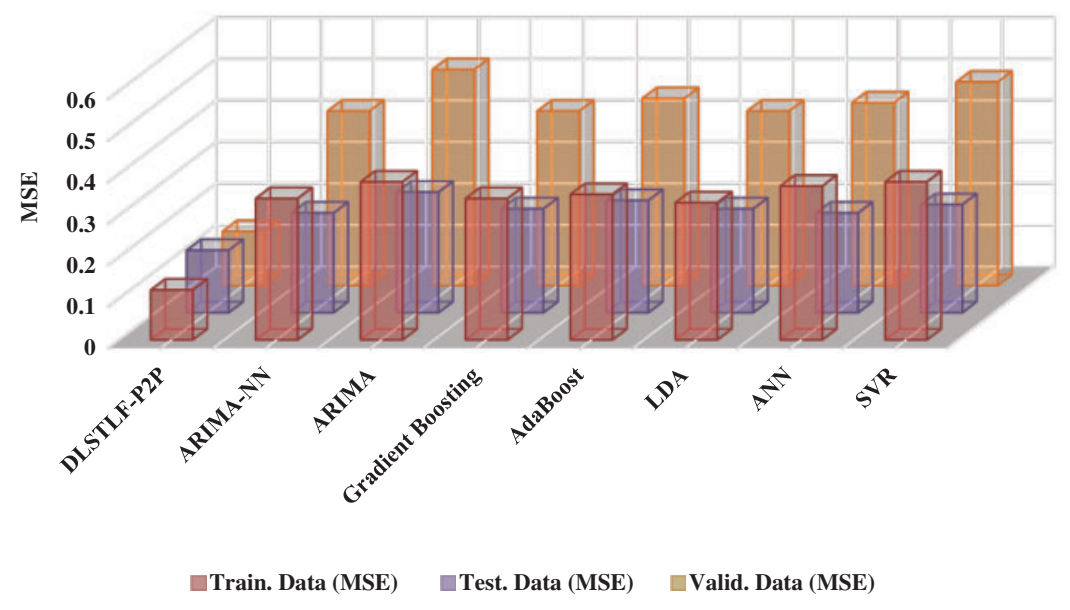

Figure 4: Result analysis of DLSTLF-P2P model interms of MSE

Table 1: Accuracy analysis of the DLSTLF-P2P technique

\begin{tabular}{llll}
\hline Methods & Train. data (Acc.) & Test. data (Acc.) & Valid. data (Acc.) \\
\hline DLSTLF-P2P & 90.17 & 87.39 & 87.86 \\
ARIMA-NN & 43.82 & 47.88 & 44.84 \\
ARIMA & 30.51 & 20.33 & 18.07 \\
Gradient Boosting & 35.65 & 40.87 & 32.78 \\
AdaBoost & 35.86 & 34.62 & 36.50 \\
LDA & 30.38 & 40.57 & 37.39 \\
ANN & 39.49 & 41.76 & 41.10 \\
SVR & 43.48 & 46.52 & 43.93 \\
\hline
\end{tabular}

Fig. 5 investigates the difference in MAPE values of the proposed with exiting techniques. On the applied validation data, it is noticed that the ARIMA and RPART techniques have exhibited higher differences in MAPE with the values of $37.10 \%$ and $25.24 \%$ respectively. In the meantime, the KNN and RF techniques have showcased slightly reduced differences in MAPE with the values of $21.68 \%$ and $19.13 \%$ respectively. Along with that, the NNET and SVR techniques have resulted in certainly lower MAPE values of $18.88 \%$ and $15.94 \%$. Moreover, the ARIMA-NN technique has depicted minimal difference in MAPE values with the proposed DLSTLF-P2P technique. 


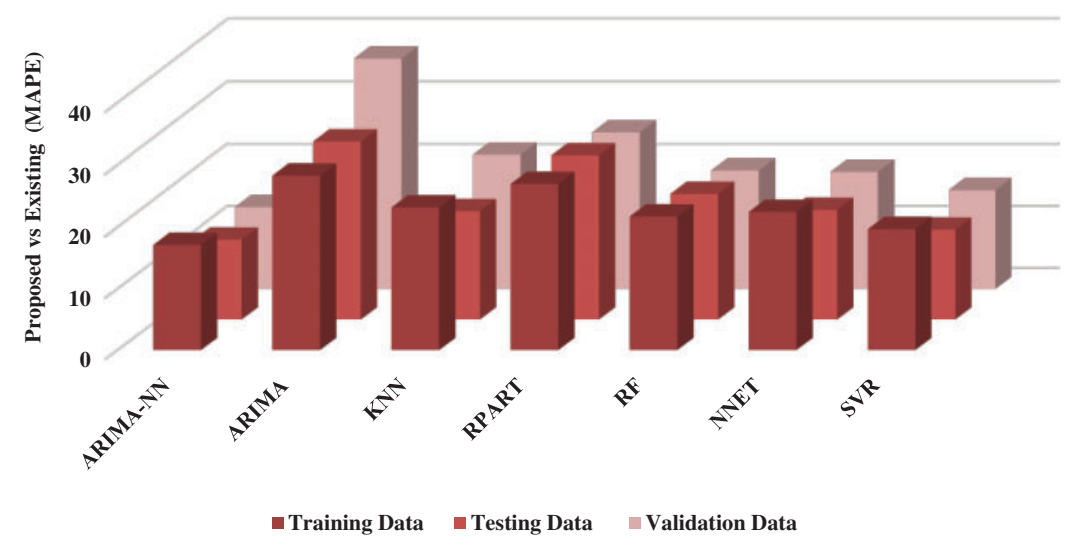

Figure 5: MAPE analysis of DLSTLF-P2P method with existing techniques

Fig. 6 examines the difference in MSE values of the projected with recent algorithms. On the applied validation data, it can be stated that the ARIMA and SVR algorithms have shown superior differences in MSE with the values of $0.39 \%$ and $0.36 \%$ correspondingly. Meanwhile, the RPART and NNET approaches have outperformed somewhat minimal differences and similar MSE with the values of $0.32 \%$ and $0.31 \%$ correspondingly. Also, the KNN and RF methods have resulted in the certainly same MSE values of $0.29 \%$. Additionally, the ARIMA-NN methodology has showcased lesser difference in MSE values with the presented DLSTLF-P2P method.

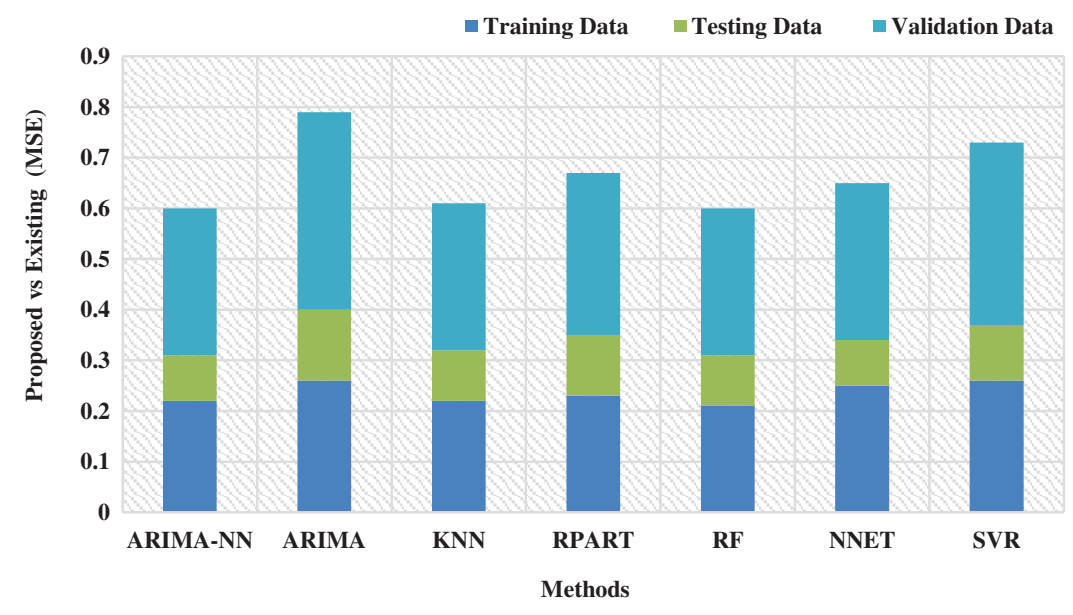

Figure 6: MSE analysis of DLSTLF-P2P method with existing techniques

Finally, Fig. 7 assesses the difference in accuracy values of the DLSTLF-P2P with existing techniques. The figure demonstrated that the ARIMA and NN techniques have showcased extreme differences in accuracy values. Followed by, the KNN and RPART techniques have gained somewhat decreased differences in accuracy values. In line with, a moderate difference in accuracy value is exhibited by the NNET technique whereas the ARIMA-NN and SVR techniques have accomplished 
lower differences in accuracy values with the proposed model. From the detailed simulation analysis, it is apparent that the DLSTLF-P2P technique has showcased effectual outcomes over the other techniques.

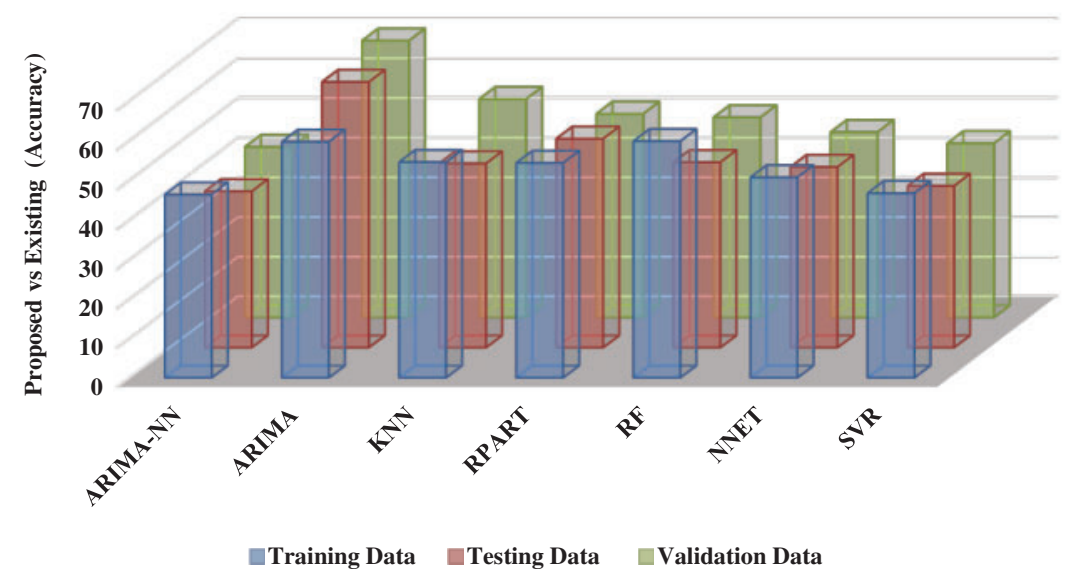

Figure 7: Accuracy analysis of DLSTLF-P2P method with existing techniques

\section{Conclusion}

This paper has introduced an efficient DLSTLF-P2P technique to trade energy in TEM. The DLSTLF-P2P technique is intended to derive effectual load forecasting model for P2P energy trading in TEM. The DLSTLF-P2P technique encompasses several processes namely pre-processing, OCOA based feature selection, DBN based classification, and CSO based hyperparameter optimization. The utilization of OCAO and CSO techniques for feature selection and parameter optimization helps to considerably enhance the predictive outcomes. A widespread experimental analysis is carried out on the benchmark UK Smart Meter dataset and inspect the results under various aspects. The experimental outcomes highlighted the promising predictive performance of the DLSTLF-P2P technique over the existing load prediction techniques. In future, the predictive outcome can be further improvised by the design of clustering and outlier detection approaches.

Funding Statement: This research was supported by Korea Institute for Advancement of Technology (KIAT) grant funded by the Korea Government (MOTIE) (P0012724, The Competency Development Program for Industry Specialist) and the Soonchunhyang University Research Fund.

Conflicts of Interest: The authors declare that they have no conflicts of interest to report regarding the present study.

\section{References}

[1] R. Dasgupta, A. Sakzad and C. Rudolph, "Cyber attacks in transactive energy market-based microgrid systems," Energies, vol. 14, no. 4, pp. 1137, 2021.

[2] S. Famila, A. Jawahar, A. Sariga and K. Shankar, "Improved artificial bee colony optimization based clustering algorithm for SMART sensor environments," Peer-to-Peer Networking and Applications, vol. 13, no. 4, pp. 1071-1079, 2020. 
[3] J. Uthayakumar, N. Metawa, K. Shankar and S. K. Lakshmanaprabu, "Intelligent hybrid model for financial crisis prediction using machine learning techniques," Information Systems and e-Business Management, vol. 18, no. 4, pp. 617-645, 2020.

[4] F. Moret and P. Pinson, "Energy collectives: A community and fairness based approach to future electricity markets," IEEE Transactions on Power Systems, vol. 34, no. 5, pp. 3994 4004, 2019.

[5] I. V. Pustokhina, D. A. Pustokhin, T. Vaiyapuri, D. Gupta, S. Kumar et al., "An automated deep learning based anomaly detection in pedestrian walkways for vulnerable road users safety," Safety Science, vol. 142, pp. 105356, 2021.

[6] K. Shankar, E. Perumal, M. Elhoseny and P. Thanh Nguyen, "An IoT-cloud based intelligent computeraided diagnosis of diabetic retinopathy stage classification using deep learning approach," Computers, Materials \& Continua, vol. 66, no. 2, pp. 1665-1680, 2021.

[7] T. Gopalakrishnan, D. Ruby, F. A. Turjman, D. Gupta, I. V. Pustokhina et al., "Deep learning enabled data offloading with cyber attack detection model in mobile edge computing systems," IEEE Access, vol. 8, pp. 185938-185949, 2020.

[8] M. A. Mohamed, A. Hajjiah, K. A. Alnowibet, A. F. Alrasheedi, E. M. Awwad et al., "A secured advanced management architecture in peer-to-peer energy trading for multi-microgrid in the stochastic environment," IEEE Access, vol. 9, pp. 92083-92100, 2021.

[9] T. Chen and $\mathrm{S} . \mathrm{Bu}$, "Realistic peer-to-peer energy trading model for microgrids using deep reinforcement learning," in 2019 IEEE PES Innovative Smart Grid Technologies Europe (ISGT-Europe), Bucharest, Romania, pp. 1-5, 2019.

[10] D. Qiu, Y. Ye, D. Papadaskalopoulos and G. Strbac, "Scalable coordinated management of peer-to-peer energy trading: A multi-cluster deep reinforcement learning approach," Applied Energy, vol. 292, no. 6, pp. 116940, 2021.

[11] F. Jamil, N. Iqbal, I. Jamal, S. Ahmad and D. Kim, "Peer-to-peer energy trading mechanism based on blockchain and machine learning for sustainable electrical power supply in smart grid," IEEE Access, vol. 9, pp. 39193-39217, 2021.

[12] M. Khorasany, Y. Mishra and G. Ledwich, "Hybrid trading scheme for peer-to-peer energy trading in transactive energy markets," IET Generation, Transmission \& Distribution, vol. 14, no. 2, pp. 245-253, 2020.

[13] M. H. Ullah, A. Alseyat and J. D. Park, "Distributed dynamic pricing in peer-to-peer transactive energy systems in smart grid," in 2020 IEEE Power \& Energy Society General Meeting (PESGM), Montreal, QC, Canada, pp. 1-5, 2020.

[14] M. Yan, M. Shahidehpour, A. Paaso, L. Zhang, A. Alabdulwahab et al., "Distribution network-constrained optimization of peer-to-peer transactive energy trading among multi-microgrids," IEEE Transactions on Smart Grid, vol. 12, no. 2, pp. 1033-1047, 2021.

[15] B. Zheng, Y. Fan, W. Wei, Y. Xu, S. Huang et al., "Distribution optimal power flow with energy sharing via a peer-to-peer transactive market," Frontiers in Energy Research, vol. 9, pp. 701149, 2021.

[16] J. G. Kim and B. Lee, "Automatic p2p energy trading model based on reinforcement learning using long short-term delayed reward," Energies, vol. 13, no. 20, pp. 5359, 2020.

[17] P. Wongthongtham, D. Marrable, B. A. Salih, X. Liu and G. Morrison, "Blockchain-enabled Peer-to-Peer energy trading," Computers \& Electrical Engineering, vol. 94, pp. 107299, 2021.

[18] M. A. Ferrag and L. Maglaras, "DeepCoin: A novel deep learning and blockchain-based energy exchange framework for smart grids," IEEE Transactions on Engineering Management, vol. 67, no. 4, pp. 1285-1297, 2020.

[19] Z. Yuan, W. Wang, H. Wang and A. Yildizbasi, "Developed coyote optimization algorithm and its application to optimal parameters estimation of PEMFC model," Energy Reports, vol. 6, no. 8, pp. 1106$1117,2020$.

[20] R. C. T. D. Souza, C. A. D. Macedo, L. D. S. Coelho, J. Pierezan and V. C. Mariani, "Binary coyote optimization algorithm for feature selection," Pattern Recognition, vol. 107, pp. 107470, 2020. 
CMC, 2022, vol.71, no.1

[21] J. Li, X. Shao and R. Sun, "A dbn-based deep neural network model with multitask learning for online air quality prediction," Journal of Control Science and Engineering, vol. 2019, no. 1, pp. 1-9, 2019.

[22] Y. Li, S. Wang and M. Han, "Truss structure optimization based on improved chicken swarm optimization algorithm," Advances in Civil Engineering, vol. 2019, no. 6, pp. 1-16, 2019.

[23] UK Smart Meter Data, 2019, https://cc-uploads.s3.amazonaws.com/open_links/HumAIn+2019/UK_Sma rt_Meter.zip. (Accessed on October 16, 2020) . 